\title{
Phase Confirmation and Equation of State of ß-HMX under 40 GPa
}

Zhilei Sui $^{\mathrm{a} \dagger}$, Xiaoyu Sun ${ }^{\mathrm{b} \dagger}$, Wentao Liang ${ }^{\mathrm{b}}$, Rucheng Dai ${ }^{\mathrm{c}}$, Zhongping Wang ${ }^{\mathrm{c}}$, Shiliang Huang ${ }^{\mathrm{d}}$, Xianxu Zheng ${ }^{\mathrm{a}^{*}}$, Zengming Zhang ${ }^{\mathrm{c}, \mathrm{e}^{*}}$, Qiang $\mathrm{Wu}^{\mathrm{a}}$

a. Institute of Fluid Physics, China Academy of Engineering Physics, Mianyang, Sichuan, 621900, China;

b. Department of Physics, University of Science and Technology of China, Hefei, Anhui 230026, China;

c. The Centre for Physical Experiments, University of Science and Technology of China, Hefei, Anhui 230026, China;

d. Institute of Chemical Materials, China Academy of Engineering Physics, Mianyang, Sichuan 621900, China

e. Key Laboratory of Strongly-Coupled Quantum Matter Physics, Chinese Academy of Sciences, School of Physical Sciences, University of Science and Technology of China, Hefei, Anhui 230026, China

†Co-authors: zlsui@mail.ustc.edu.cn, xysun2015@mail.ustc.edu.cn

*Corresponding authors: zzm@ustc.edu.cn Phone: +8655163607671; zxxgoal109@sina.com Phone: +868162485106 . 
Table S1 Frequencies of Raman modes and infrared modes of HMX.

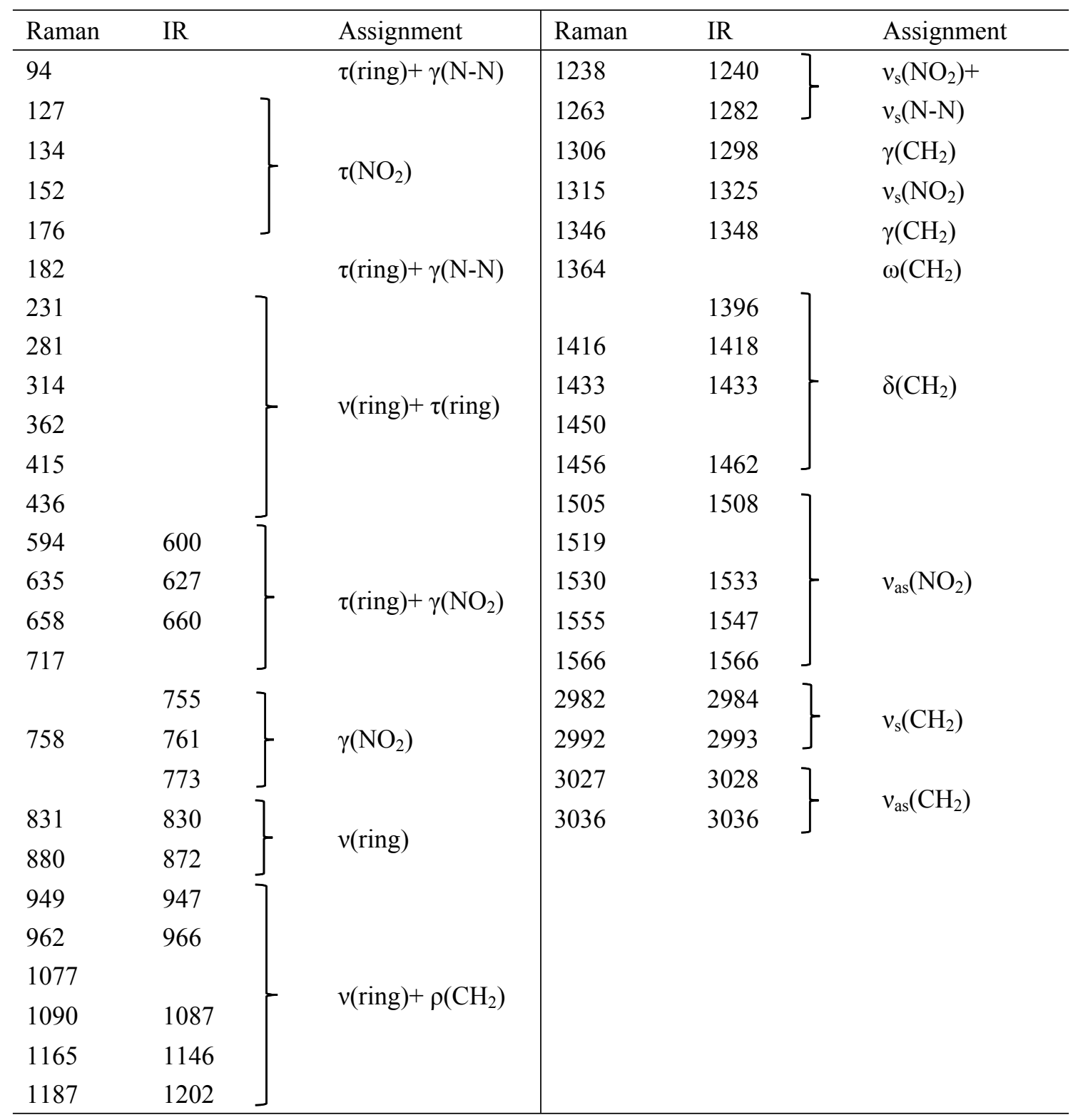

as: anti-symmetric; s: symmetric; $v$ : stretching; $\delta$ : deformation motion; $\tau$ : torsional motion; $\gamma$ : deformations involving one ring and one non-ring bond; $\omega\left(\mathrm{XY}_{2}\right)$ : wag of $\mathrm{Y}_{2}$ atoms out of $\mathrm{XY}_{2}$ plane; $\rho\left(X_{2}\right)$ : rocking in $X Y_{2}$ plane. 
Table S2 The slope of pressure and Raman mode frequencies.

\begin{tabular}{|c|c|c|c|c|c|c|c|c|c|c|c|}
\hline & $\beta$ & $\zeta$ & $\varepsilon$ & $\varphi$ & $\eta$ & & $\beta$ & $\zeta$ & $\varepsilon$ & $\varphi$ & $\eta$ \\
\hline$v_{1}$ & & & 1.5 & 1.6 & 1.7 & $v_{39}$ & & 0.9 & 0.7 & 0.7 & 0.9 \\
\hline$v_{2}$ & & & 2.1 & & & $v_{40}$ & 4.4 & 3.8 & 3.0 & 2.5 & 2.2 \\
\hline$v_{3}$ & & 3.2 & 1.7 & 1.3 & 1.3 & $v_{41}$ & 3.6 & 3.0 & 2.7 & 2.6 & 1.5 \\
\hline$v_{4}$ & 4.7 & 3.1 & & & & $v_{42}$ & & & 1.3 & 1.0 & 1.5 \\
\hline$v_{5}$ & 5.4 & 4.0 & 2.5 & 1.7 & 1.3 & $v_{43}$ & & & & 1.5 & 1.2 \\
\hline$v_{6}$ & 7.5 & 4.2 & 2.3 & 1.4 & 1.1 & $v_{44}$ & 4.9 & 3.4 & 2.4 & 2.0 & 1.5 \\
\hline$v_{7}$ & & & 3.4 & 2.5 & & $v_{45}$ & 4.0 & 3.1 & 2.7 & & \\
\hline$v_{8}$ & 6.9 & 3.5 & 2.1 & 2.2 & 1.3 & $v_{46}$ & 6.3 & & & & \\
\hline$v_{9}$ & & & & 2.6 & 1.9 & $v_{47}$ & 6.6 & 4.9 & 3.3 & 2.3 & 1.5 \\
\hline$v_{10}$ & & & 3.6 & 3.2 & 2.4 & $v_{48}$ & 7.5 & 4.7 & 3.1 & & \\
\hline$v_{11}$ & 9.7 & 6.2 & 4.6 & & & $v_{49}$ & 3.2 & 3.1 & 2.5 & 1.9 & 1.0 \\
\hline$v_{12}$ & 10.8 & 5.5 & 2.9 & 2.4 & 2.1 & $v_{50}$ & 3.7 & 3.1 & 2.4 & & \\
\hline$v_{13}$ & 10.5 & 6.4 & 3.7 & 2.2 & 2.3 & $v_{51}$ & 5.1 & 4.8 & 3.1 & 1.8 & 1.1 \\
\hline$v_{14}$ & & & & 3.5 & 2.2 & $v_{52}$ & 3.4 & 3.0 & 2.7 & 2.4 & \\
\hline$v_{15}$ & & & & & 2.7 & $v_{53}$ & & 3.7 & 3.0 & 3.2 & \\
\hline$v_{16}$ & 7.5 & 7.1 & 4.5 & 3.1 & & $v_{54}$ & 3.2 & 3.6 & 2.9 & & \\
\hline$v_{17}$ & 4.8 & 4.4 & 4.1 & 3.2 & 1.6 & $v_{55}$ & 4.2 & & & & \\
\hline$v_{18}$ & 3.7 & 2.8 & 2.6 & 2.0 & 2.1 & $v_{56}$ & 4.2 & 1.9 & 1.4 & 2.1 & 2.5 \\
\hline$v_{19}$ & & 3.7 & 2.8 & 2.6 & 2.3 & $v_{57}$ & 2.3 & 2.7 & 2.7 & 1.6 & 1.6 \\
\hline$v_{20}$ & & & & & 0.7 & $v_{58}$ & 2.0 & 2.0 & 1.6 & 1.8 & 1.5 \\
\hline$v_{21}$ & 3.4 & 2.6 & 2.4 & 3.0 & 3.0 & $v_{59}$ & -0.3 & 1.2 & 0.9 & 1.7 & \\
\hline$v_{22}$ & 5.4 & 3.5 & 3.0 & 3.0 & 3.0 & $v_{60}$ & 2.0 & & & & \\
\hline$v_{23}$ & 5.3 & 3.9 & 2.9 & 2.1 & 0.8 & $v_{61}$ & & & 2.0 & 1.7 & 3.2 \\
\hline$v_{24}$ & 4.0 & 2.7 & 2.4 & 2.5 & 1.8 & $v_{62}$ & & & & & 2.7 \\
\hline$v_{25}$ & 2.0 & 1.8 & 1.7 & & & $v_{63}$ & 2.0 & 1.9 & 1.9 & 2.5 & 2.2 \\
\hline$v_{26}$ & 2.9 & 2.3 & 2.5 & 2.7 & 1.5 & $v_{64}$ & 3.2 & 2.8 & 2.4 & & \\
\hline$v_{27}$ & & 2.8 & 2.0 & 1.3 & 1.7 & $v_{65}$ & 1.1 & 0.5 & 0 & 0.9 & \\
\hline$v_{28}$ & 3.9 & 3.6 & 2.3 & 1.8 & 1.4 & $v_{66}$ & 1.5 & 1.6 & 0.8 & 0.9 & 0.9 \\
\hline$v_{29}$ & 3.9 & 2.8 & 1.8 & 1.2 & 0.7 & $v_{67}$ & 2.2 & & & & \\
\hline$v_{30}$ & & & & & 1.5 & $v_{68}$ & & & 1.7 & 1.4 & 0.7 \\
\hline$v_{31}$ & 2.8 & 2.4 & 2.0 & 1.8 & 0.9 & $v_{69}$ & 0.6 & 2.3 & 1.8 & 2.5 & 1.4 \\
\hline$v_{32}$ & & & & & 0.6 & $v_{70}$ & 0.2 & 1.4 & 2.4 & 2.7 & 1.9 \\
\hline$v_{33}$ & 1.7 & 1.5 & 1.2 & 0.6 & 0.2 & $v_{71}$ & 7.9 & 4.9 & 3.2 & 2.1 & 1.7 \\
\hline$v_{34}$ & & & & & -0.4 & $v_{72}$ & & & & 4.5 & 2.0 \\
\hline$v_{35}$ & & 0.3 & 0 & 0.3 & 0.8 & $v_{73}$ & 8.4 & 6.4 & 4.8 & 3.6 & 1.7 \\
\hline$v_{36}$ & 1.2 & 1.0 & 0.5 & 0.4 & 0.2 & $v_{74}$ & 7.1 & 4.3 & 3.9 & 3.9 & 3.0 \\
\hline$v_{37}$ & & & & & 0.2 & $v_{75}$ & 8.1 & 6.9 & 5.3 & 4.3 & 2.8 \\
\hline$v_{38}$ & & & & & 0.9 & $v_{76}$ & & & & 4.8 & 3.8 \\
\hline
\end{tabular}


Table S3 The pressure dependence of HMX crystal structure in hydrostatic conditions

\begin{tabular}{cccccc}
\hline $\begin{array}{c}\text { Pressure } \\
(\mathrm{GPa})\end{array}$ & $V(\AA)$ & $a(\AA)$ & $b(\AA)$ & $c(\AA)$ & $\beta\left({ }^{\circ}\right)$ \\
\hline 0 & 519.24 & 6.543 & 11.049 & 8.708 & 124.43 \\
1.5 & 485.82 & 6.481 & 10.618 & 8.628 & 125.08 \\
1.9 & 472.58 & 6.429 & 10.492 & 8.576 & 125.22 \\
3.2 & 454.86 & 6.360 & 10.307 & 8.526 & 125.52 \\
4.6 & 440.44 & 6.253 & 10.240 & 8.449 & 125.49 \\
8.5 & 411.32 & 6.118 & 9.854 & 8.363 & 125.33 \\
11.3 & 402.87 & 6.100 & 9.705 & 8.361 & 125.53 \\
12.2 & 398.56 & 6.099 & 9.556 & 8.404 & 125.54 \\
13.8 & 394.27 & 6.084 & 9.476 & 8.400 & 125.49 \\
15.8 & 384.84 & 5.992 & 9.427 & 8.308 & 124.90 \\
20.5 & 368.72 & 5.933 & 8.991 & 8.393 & 124.56 \\
21.6 & 364.60 & 5.902 & 8.914 & 8.405 & 124.45 \\
24.0 & 358.38 & 5.874 & 8.784 & 8.408 & 124.30 \\
29.3 & 351.81 & 5.833 & 8.702 & 8.359 & 124.00 \\
35.5 & 344.19 & 5.807 & 8.570 & 8.317 & 124.13 \\
38.0 & 336.53 & 5.765 & 8.486 & 8.242 & 123.84 \\
\hline
\end{tabular}




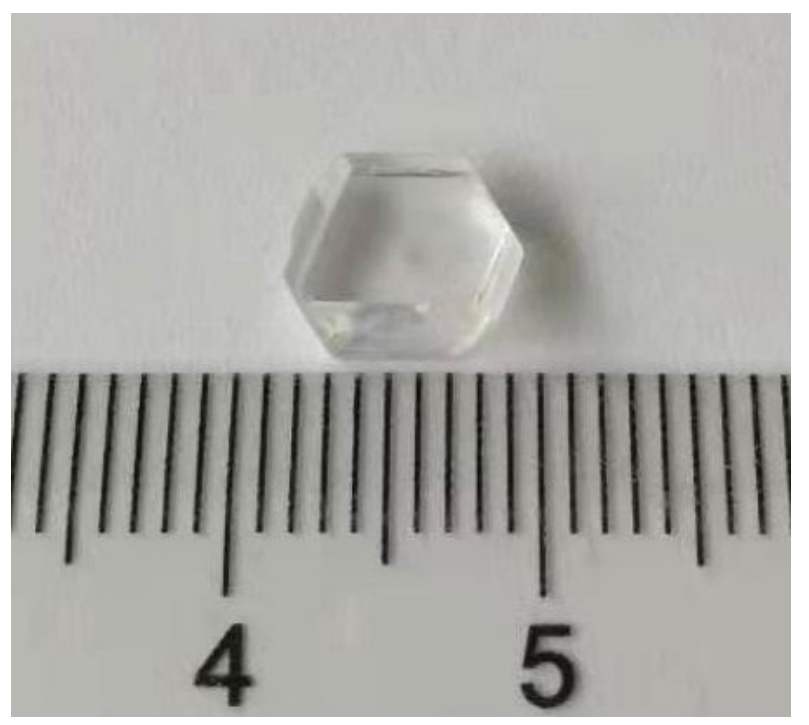

Figure S1 Photograph of $\beta-$ HMX single crystal. 


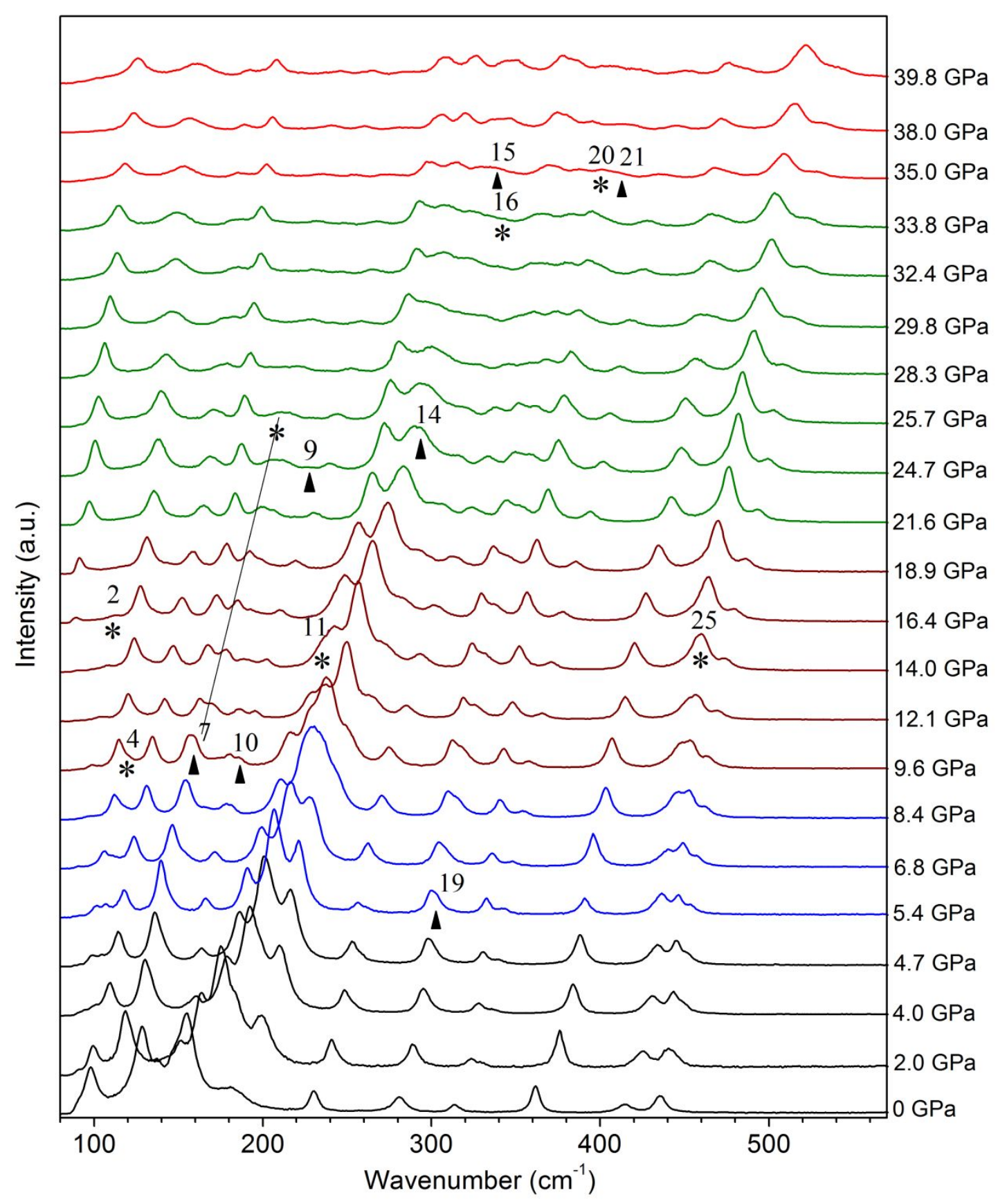

Figure S2 Raman spectra of HMX crystals under hydrostatic pressures in the range of 80-600 $\mathrm{cm}^{-1}$ (' $\boldsymbol{\Delta}$ ' represents the appearance of new peak; '*' represents the disappearance of peak). 


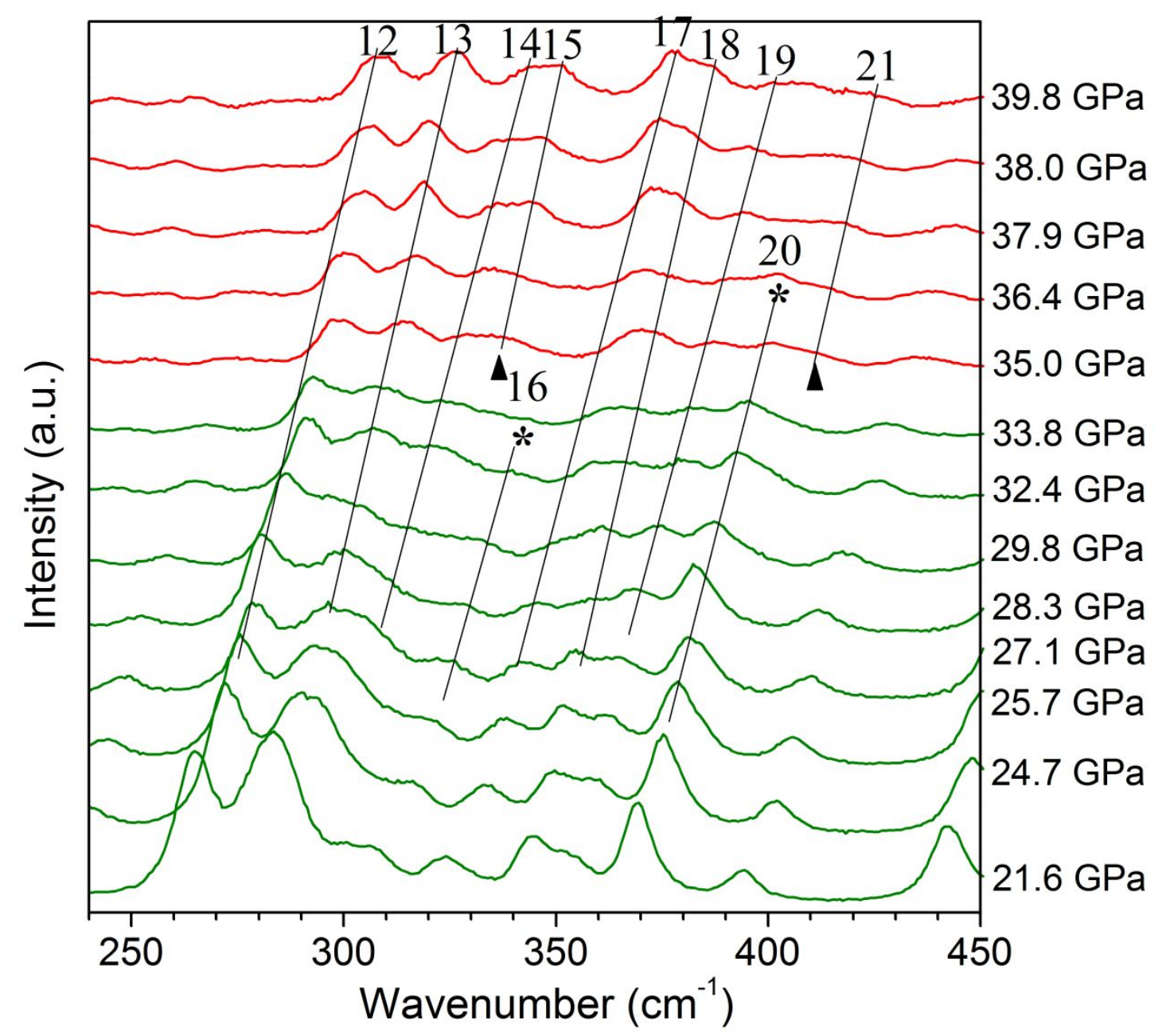

Figure S3 Raman spectra of HMX crystals under hydrostatic pressures in the range of $240-450 \mathrm{~cm}^{-1}$ (' $\boldsymbol{\Delta}$ ' represents the appearance of new peak; ' $*$ ' represents the disappearance of peak). 


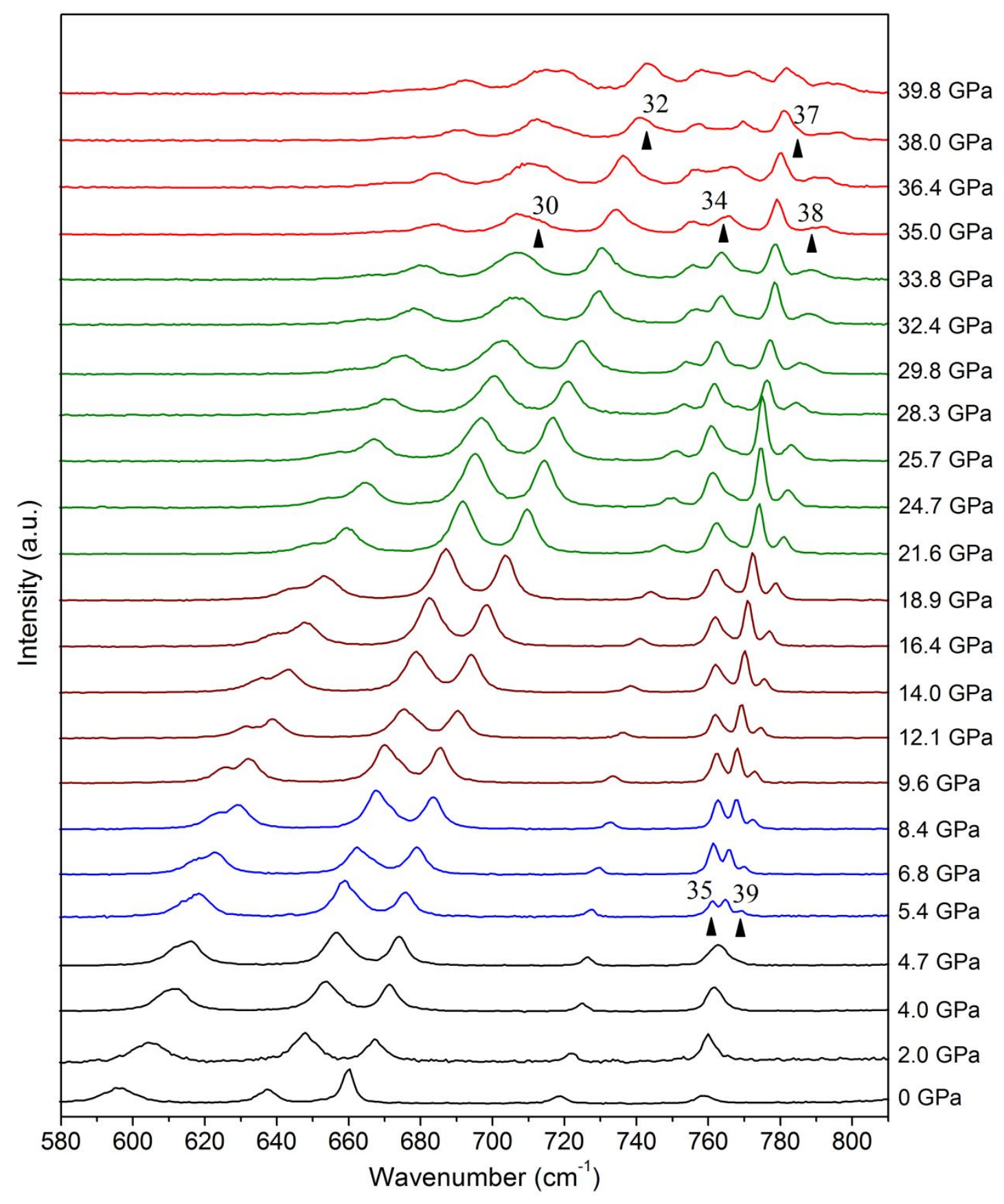

Figure S4 Raman spectra of HMX crystals under hydrostatic pressures in the range of $580-820 \mathrm{~cm}^{-1}$ (' $\boldsymbol{\Delta}$ ' represents the appearance of new peak). 


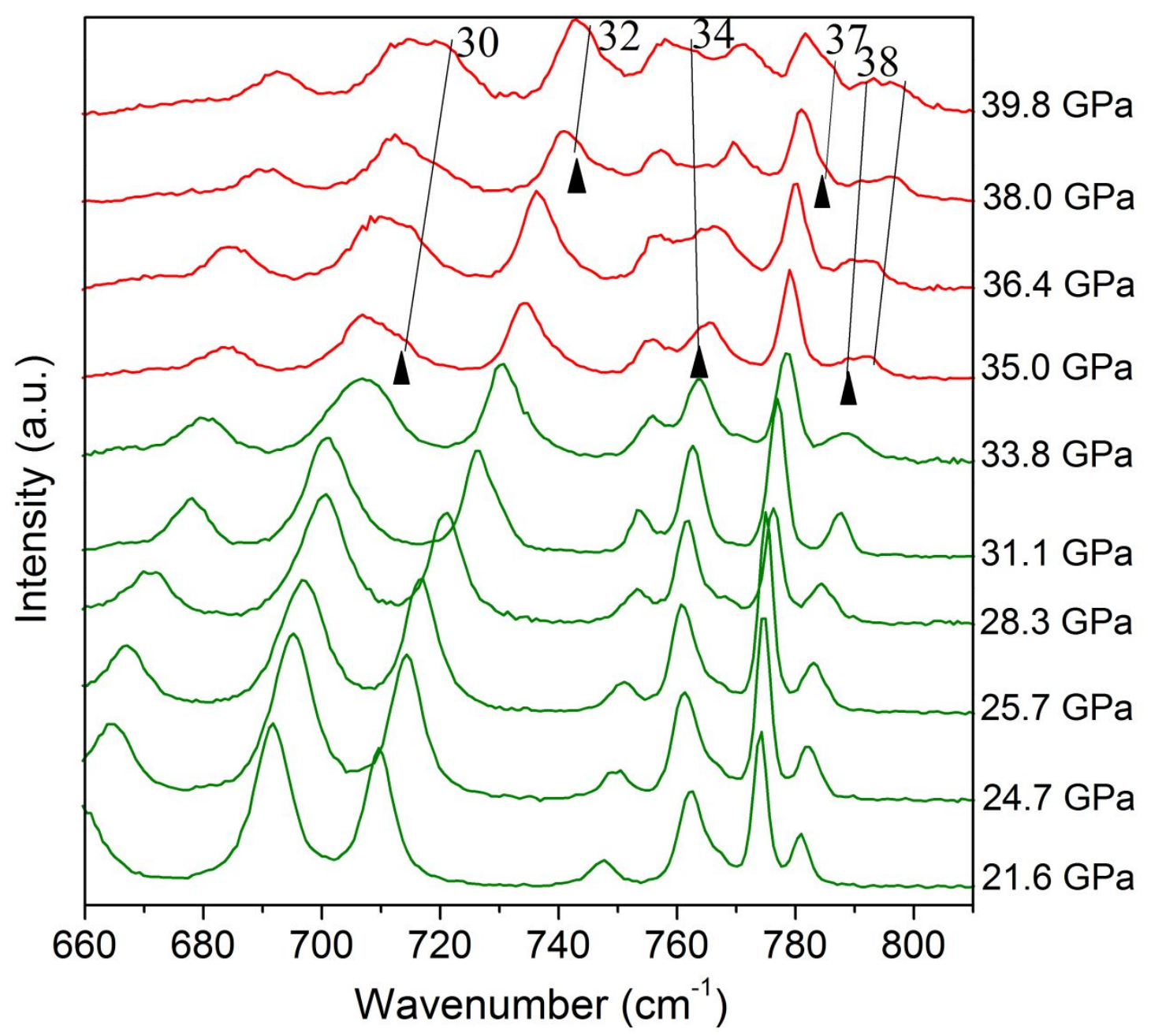

Figure S5 Raman spectra of HMX crystals under hydrostatic pressures in the range of $660-810 \mathrm{~cm}^{-1}$ (' $\boldsymbol{\Delta}$ ' represents the appearance of new peak). 


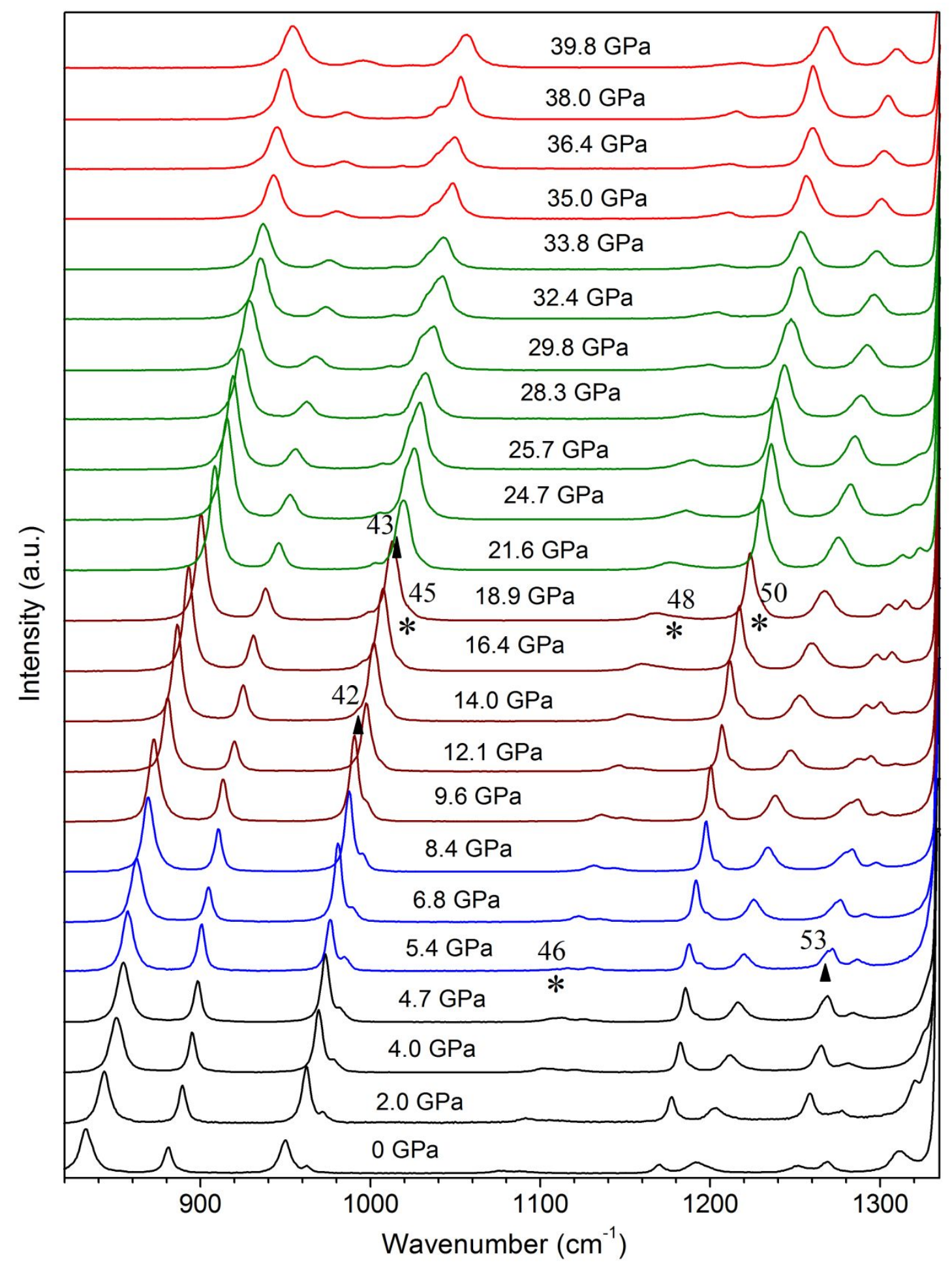

Figure S6 Raman spectra of HMX crystals under hydrostatic pressures in the range of 820-1330 $\mathrm{cm}^{-1}$ (' $\boldsymbol{\Delta}$ ' represents the appearance of new peak; '*' represents the disappearance of peak). 


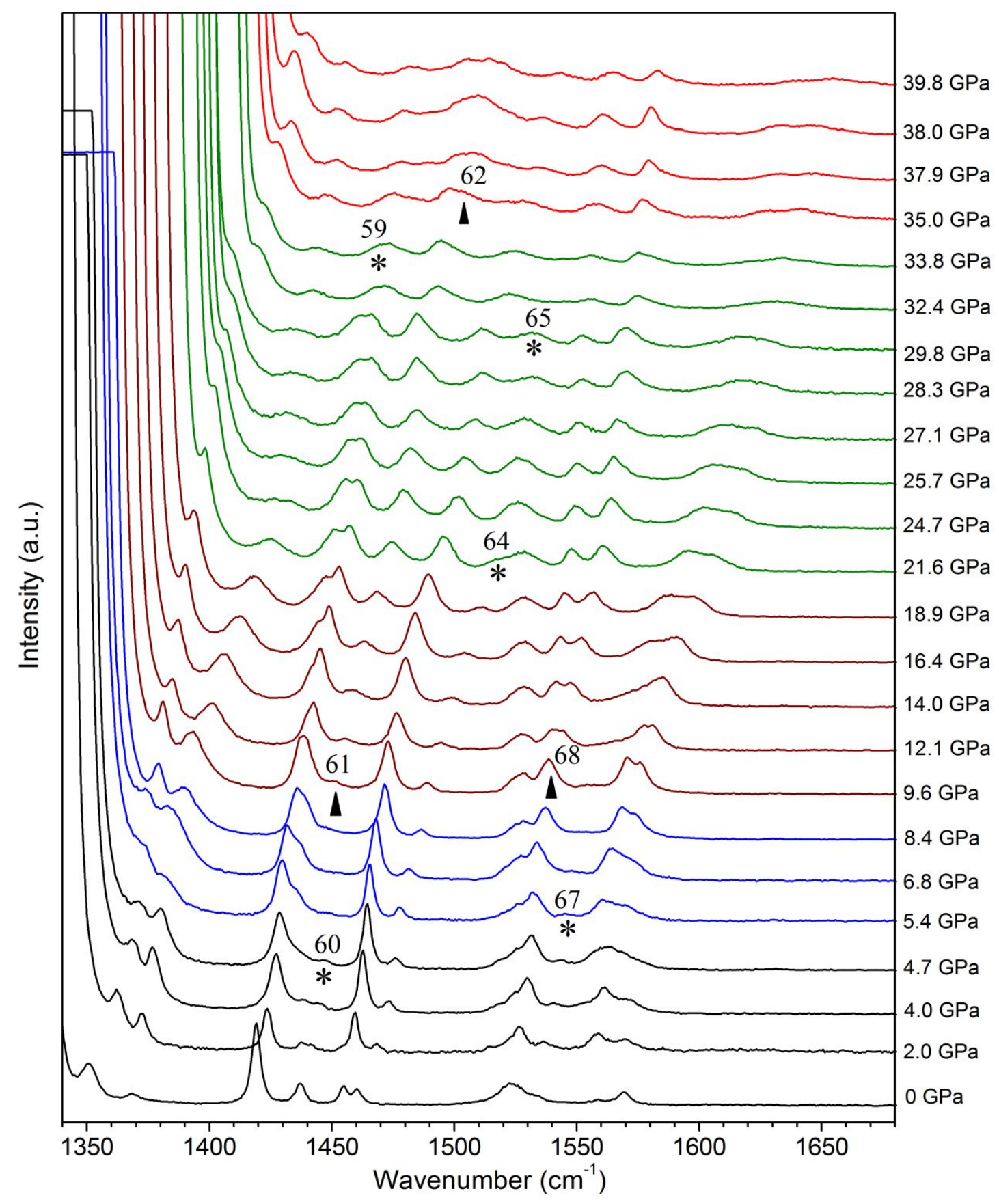

Figure S7 Raman spectra of HMX crystals under hydrostatic pressures in the range of 1340-1680 $\mathrm{cm}^{-1}$ (' $\boldsymbol{\Delta}$ ' represents the appearance of new peak; '*' represents the disappearance of peak). 


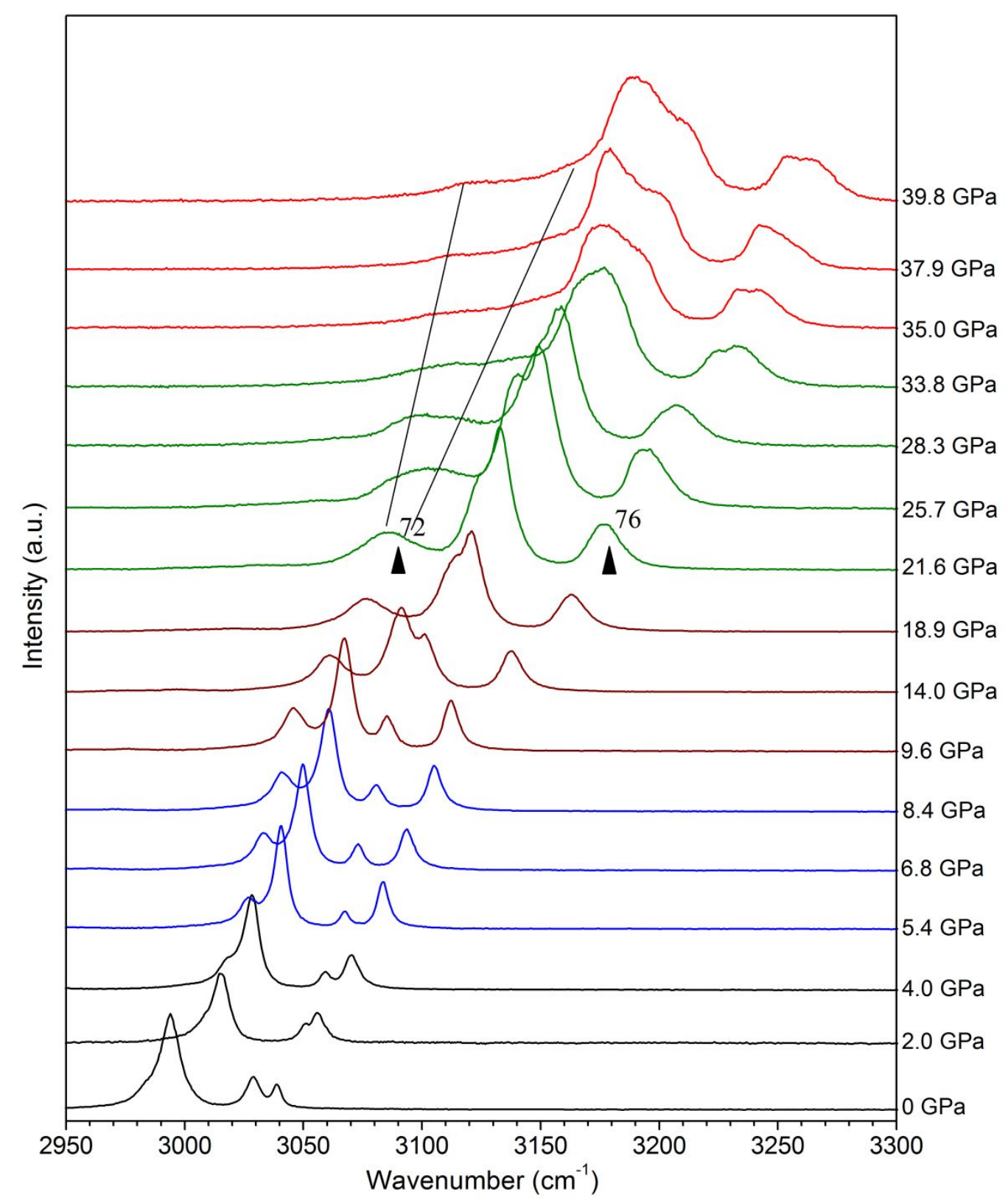

Figure S8 Raman spectra of HMX crystals under hydrostatic pressures in the range of 2950-3300 $\mathrm{cm}^{-1}$ (' $\boldsymbol{\Delta}$ ' represents the appearance of new peak). 


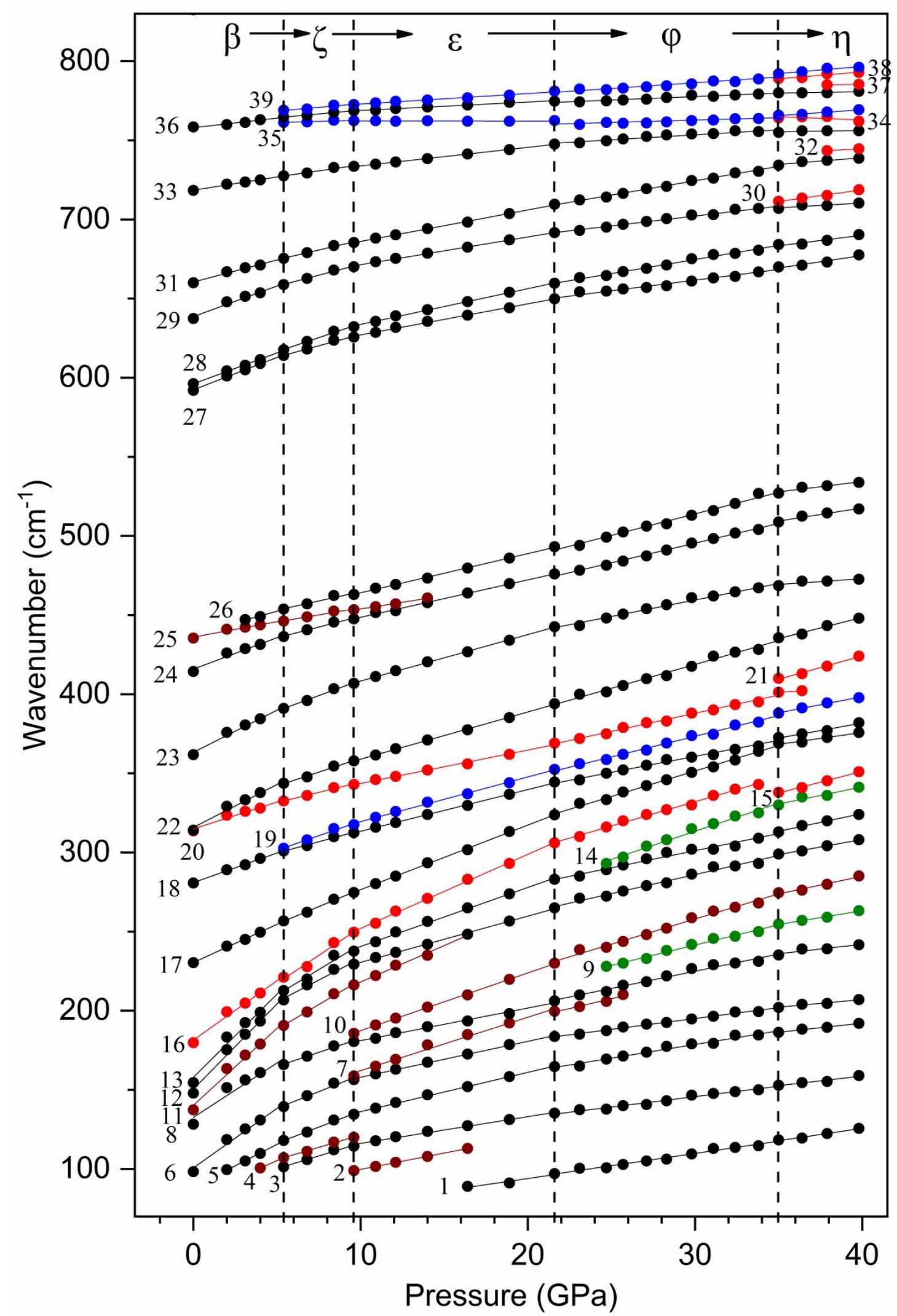

Figure S9 Frequencies of Raman Modes of HMX at different pressures in the range of $80-800 \mathrm{~cm}^{-1}$. 


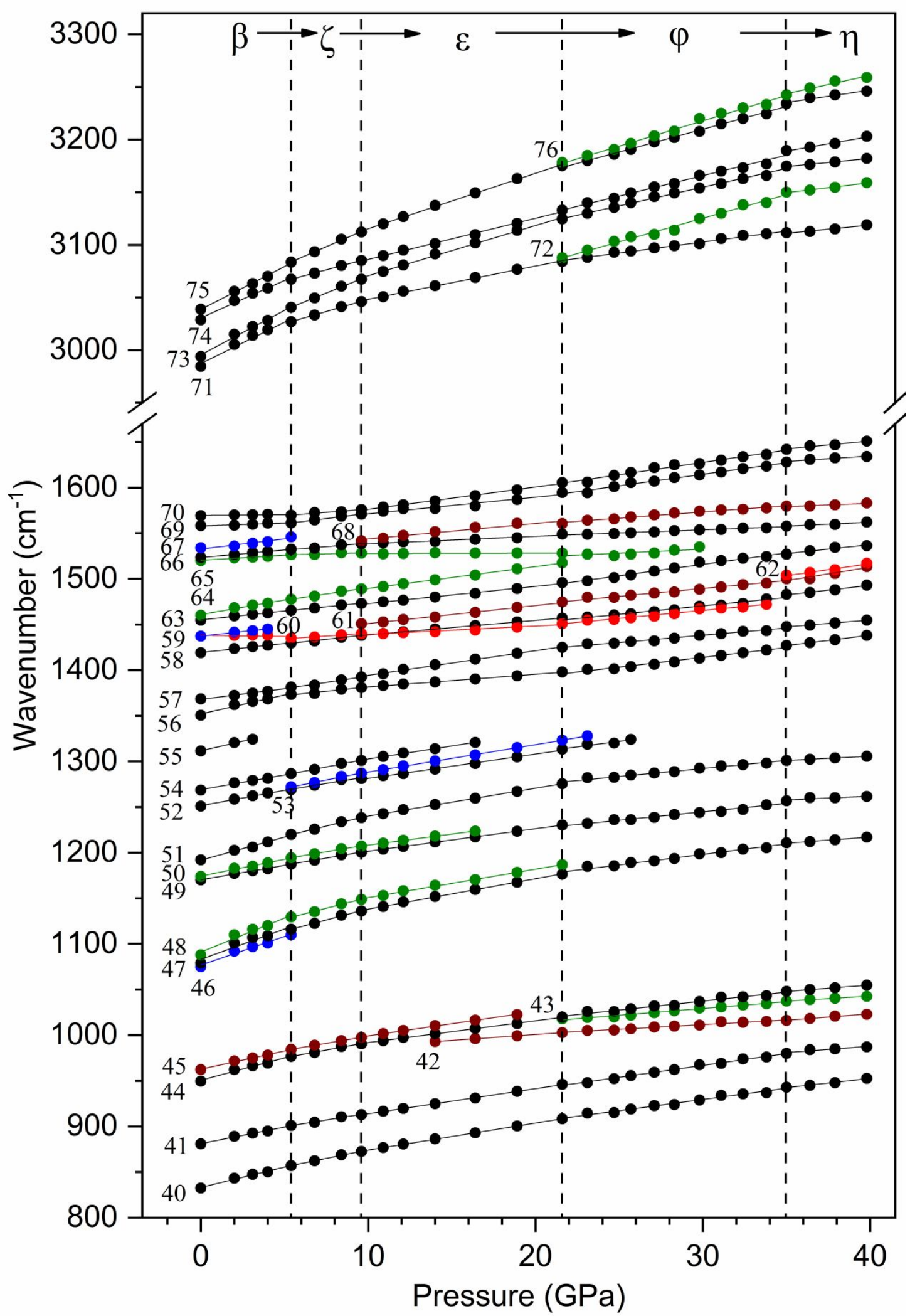

Figure S10 Frequencies of Raman Modes of HMX at different pressures in the range of $800-3300 \mathrm{~cm}^{-1}$. 
As shown in Figs. S2-S10 and Tab. S2, the changes are including:

1. $\beta \rightarrow \zeta$ phase transition around $5.4 \mathrm{GPa}$

(i) Appearance: $v_{19}, v_{35}, v_{39}$ and $v_{53}$; (ii) Disappearance: $v_{46}, v_{60}$ and $v_{67}$; (iii) Discontinuity: $v_{6}, v_{8}, v_{11}, v_{12}, v_{13}, v_{22}, v_{56}, v_{59}, v_{69}, v_{70}, v_{71}, v_{73}$ and $v_{74}$.

2. $\zeta \rightarrow \varepsilon$ phase transition around $9.6 \mathrm{GPa}$

(i) Appearance: $v_{7}, v_{10}, v_{61}$ and $v_{68}$; (ii) Disappearance: $v_{4}$; (iii) Discontinuity: $v_{3}, v_{5}$, $v_{6}, v_{12}, v_{13}, v_{27}, v_{28}, v_{29}, v_{47}, v_{48}, v_{49}, v_{50}, v_{51}, v_{69}, v_{70}, v_{71}, v_{73}$ and $v_{75}$.

3. $\varepsilon \rightarrow \varphi$ phase transition around $21.6 \mathrm{GPa}$

(i) Appearance: $v_{9}, v_{14}, v_{43}, v_{72}$ and $v_{76}$; (ii) Disappearance: $v_{45}, v_{48}, v_{50}$ and $v_{64}$; (iii) Discontinuity: $v_{5}, v_{6}, v_{7}, v_{13}, v_{16}, v_{27}, v_{28}, v_{29}, v_{47}, v_{49}, v_{51}, v_{56}, v_{57}$ and $v_{59}$.

4. $\varphi \rightarrow \eta$ phase transition around $35.0 \mathrm{GPa}$

(i) Appearance: $v_{15}, v_{21}, v_{30}, v_{32}, v_{34}, v_{37}, v_{38}$ and $v_{62}$; (ii) Disappearance: $v_{16}, v_{20}$ and $v_{59}$; (iii) Discontinuity: $v_{17}, v_{23}, v_{24}, v_{26}, v_{61}, v_{72}, v_{73}, v_{74}$ and $v_{75}$. 


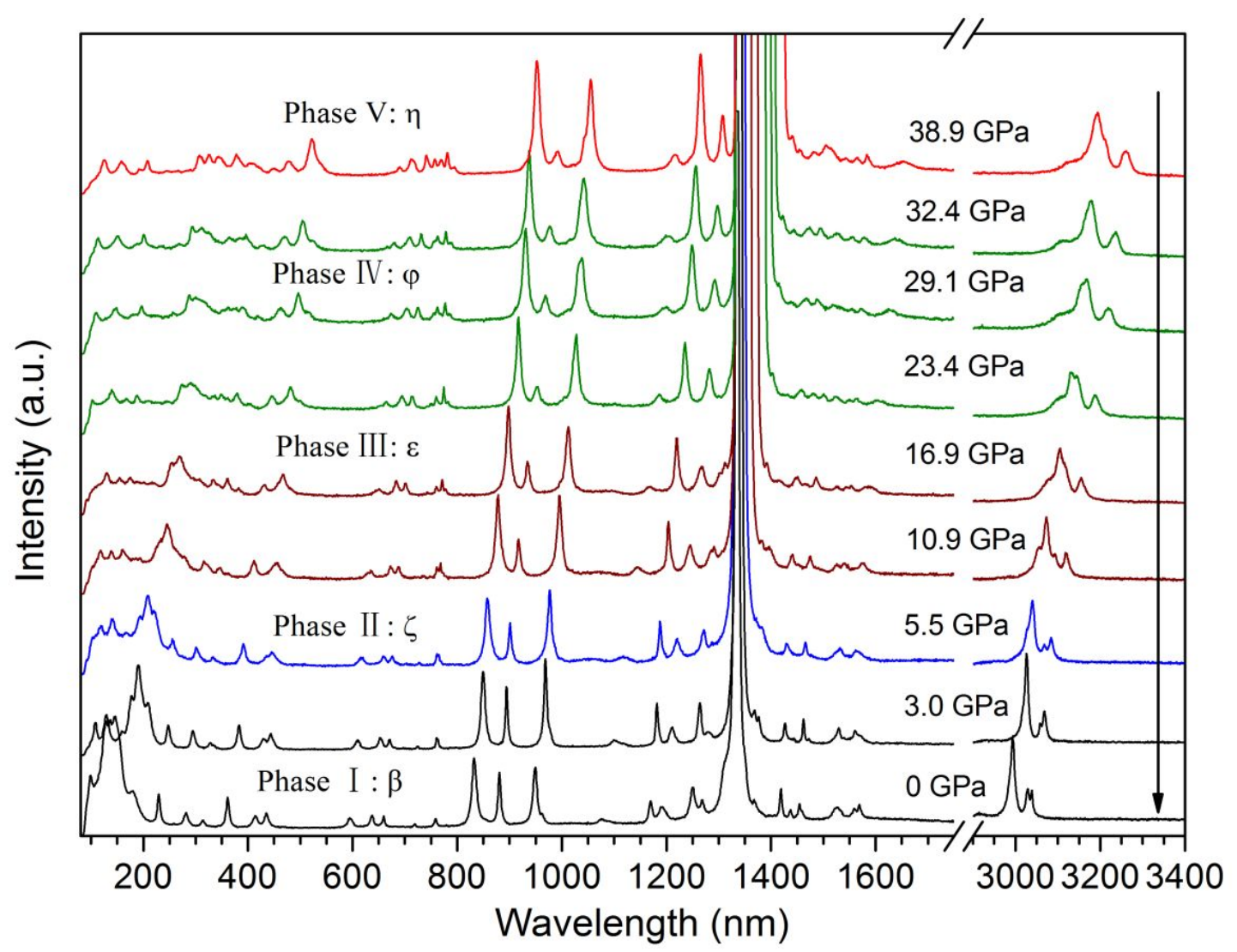

Figure S11 Raman spectra of HMX crystals under hydrostatic pressures in the process of unloading pressure.

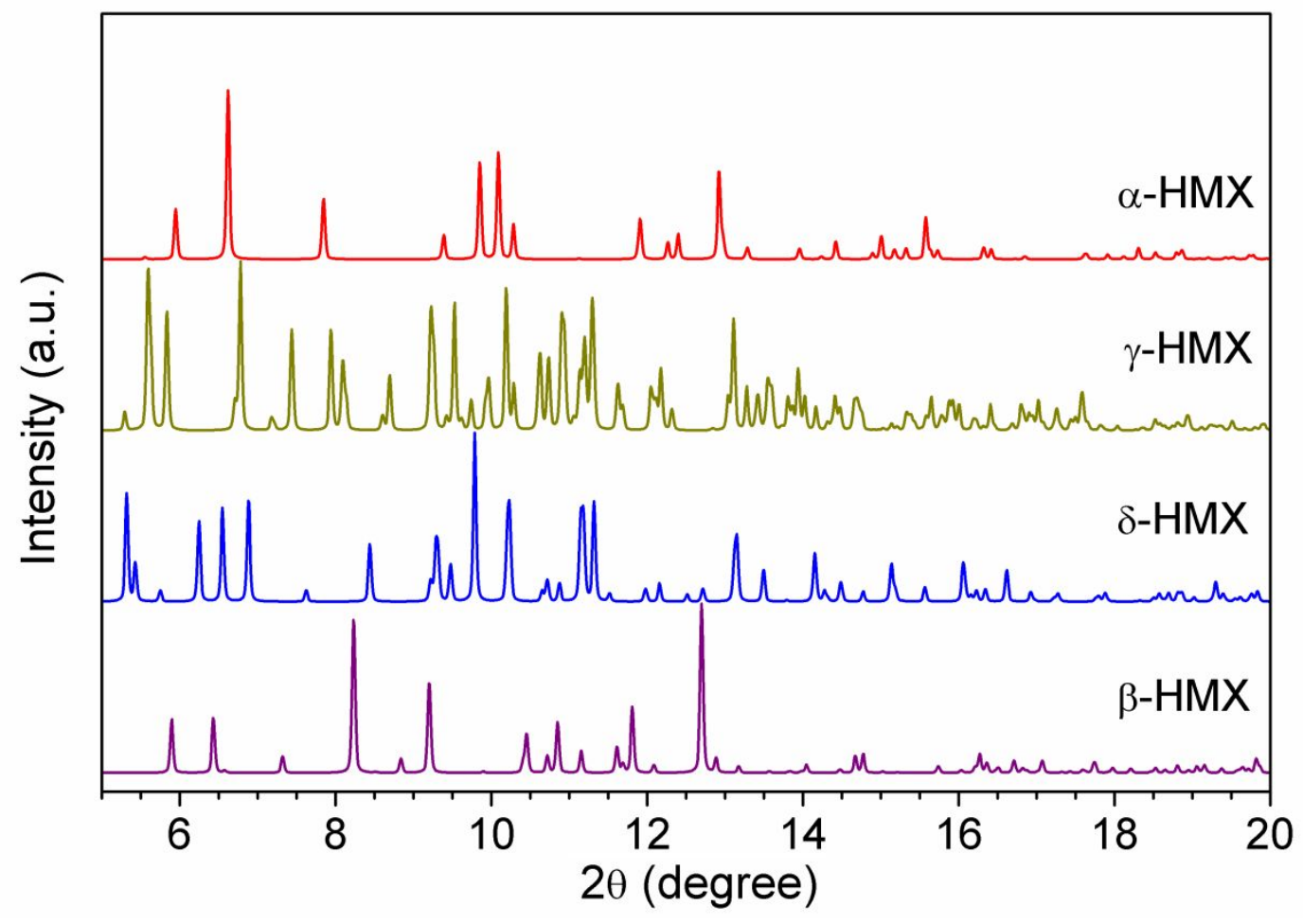

Figure S12 X-ray diffraction patterns of $\alpha-, \gamma-, \delta$ - and $\beta$-HMX. 


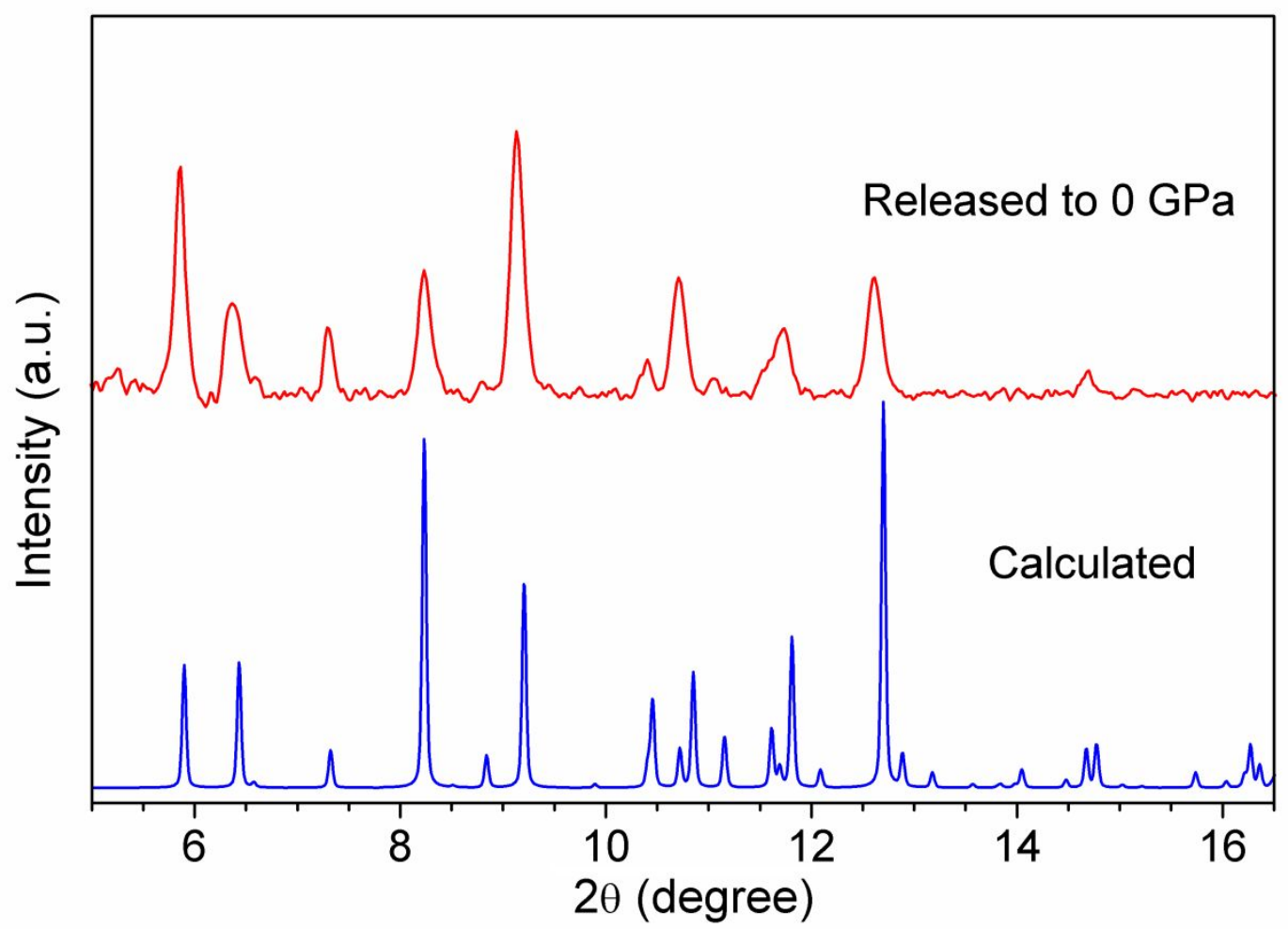

Figure S13 Synchrotron X-ray diffraction spectra of HMX after releasing pressure with neon pressure medium.

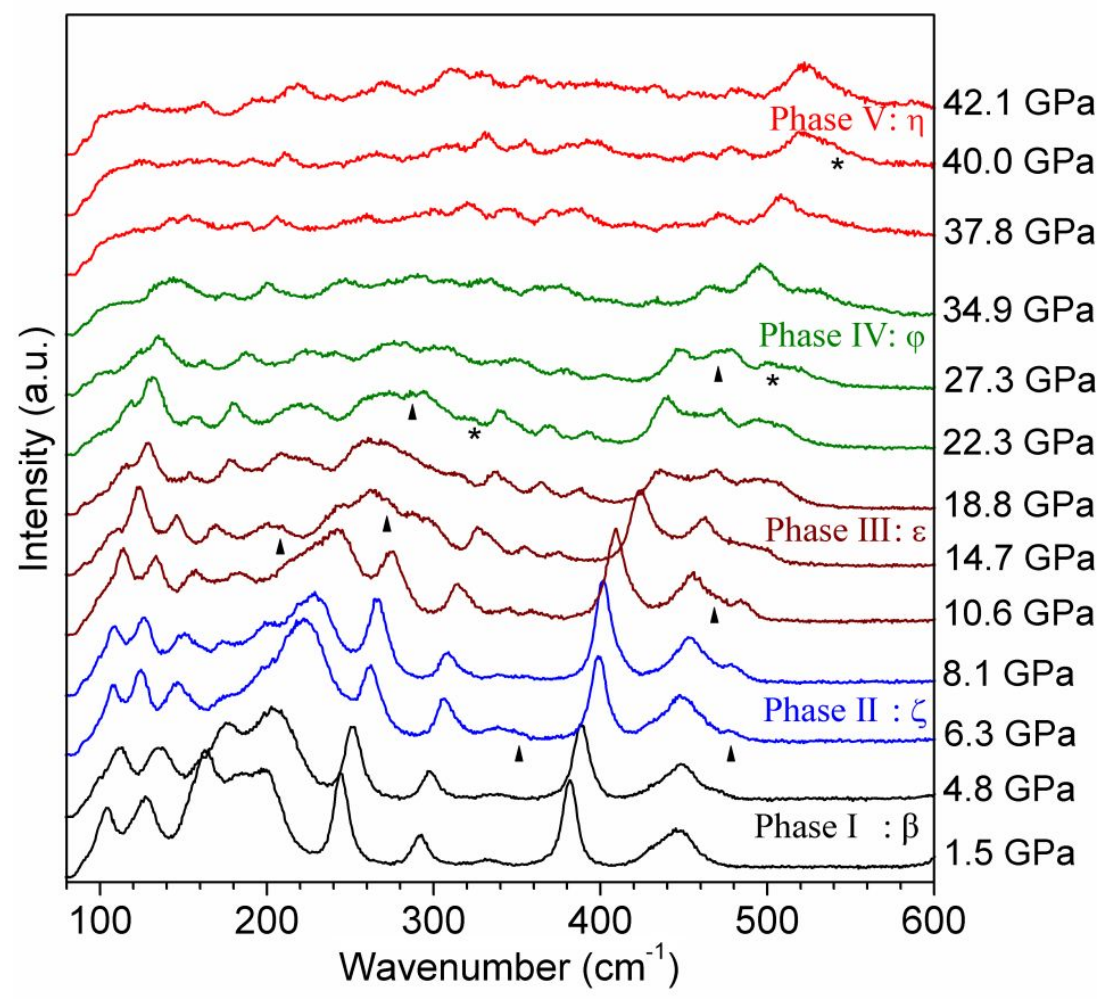

Figure S14 Raman spectra of HMX under non-hydrostatic pressures in the range of $100-600 \mathrm{~cm}^{-1}$ (' $\boldsymbol{\Delta}$ ' represents the appearance of new peak; '*' represents the disappearance of peak). 


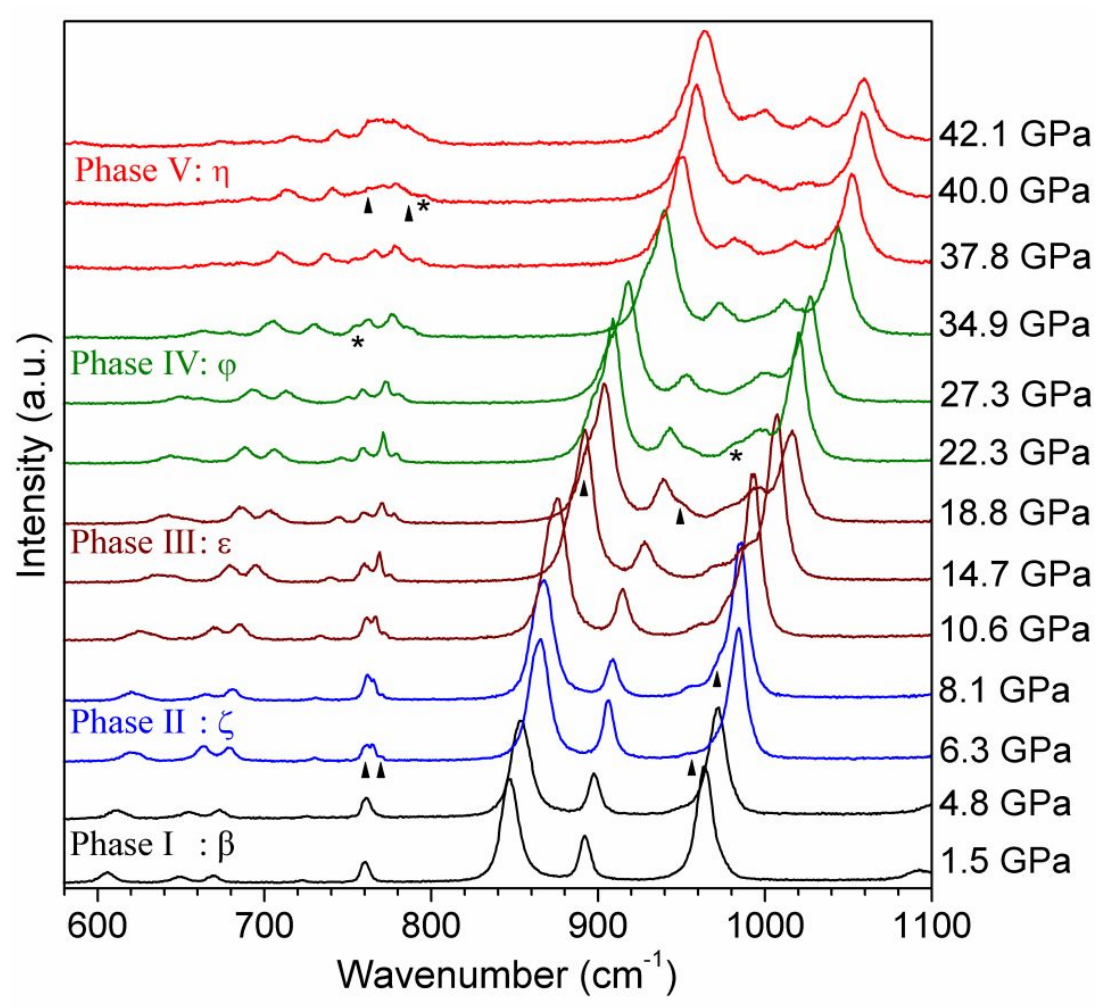

Figure S15 Raman spectra of HMX under non-hydrostatic pressures in the range of 600-1100 $\mathrm{cm}^{-1}$ (' $\boldsymbol{\Delta}$ ' represents the appearance of new peak; '*' represents the disappearance of peak).

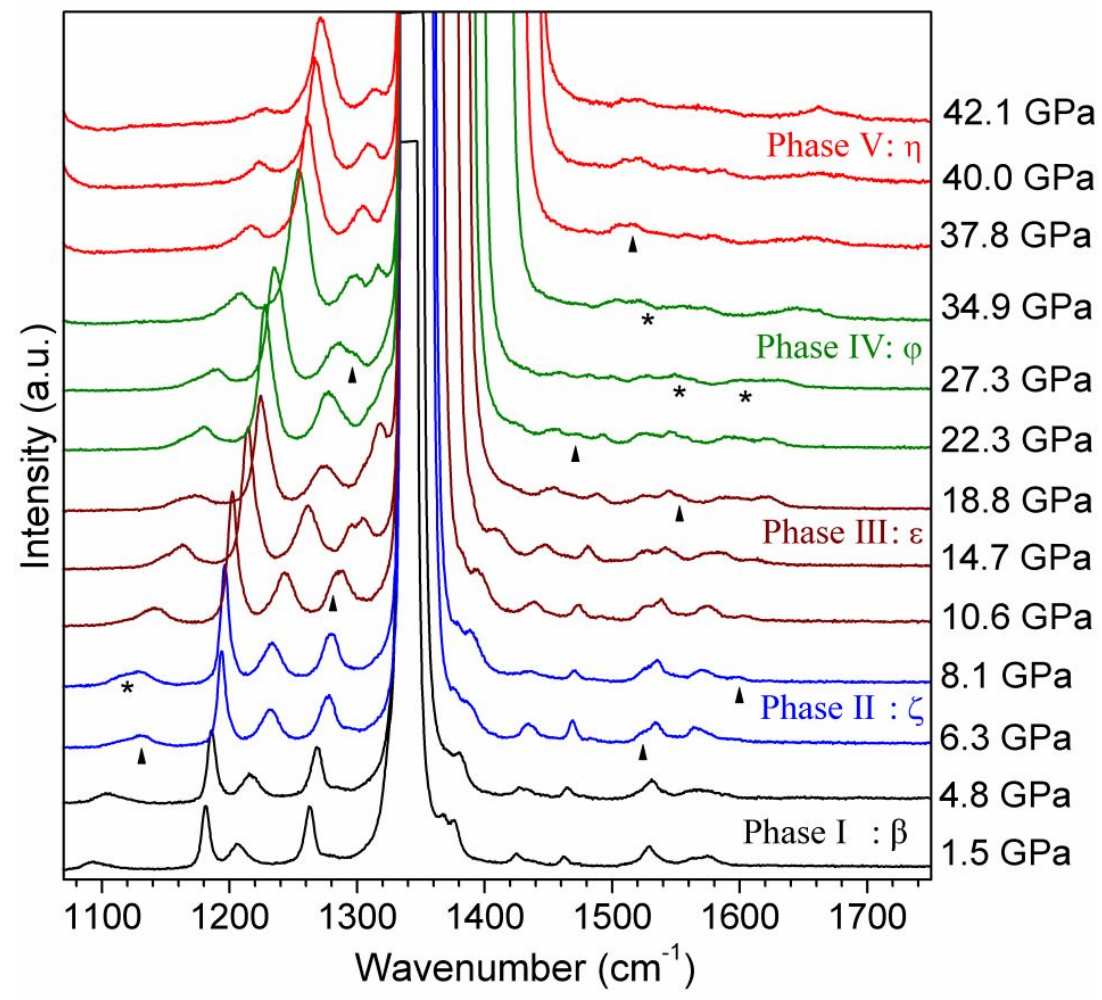

Figure S16 Raman spectra of HMX under non-hydrostatic pressures in the range of 1100-1700 $\mathrm{cm}^{-1}$ (' $\boldsymbol{\Delta}$ ' represents the appearance of new peak; '*' represents the disappearance of peak). 


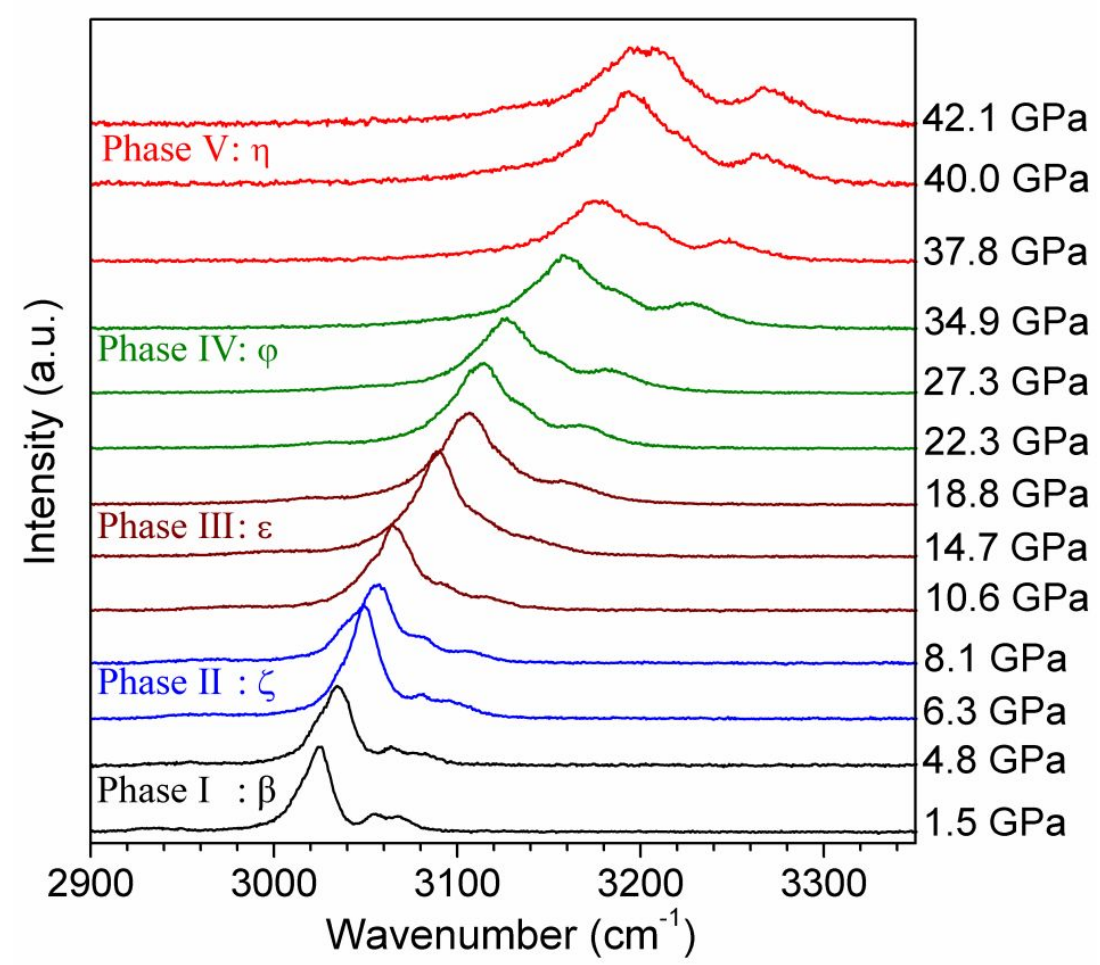

Figure S17 Raman spectra of HMX under non-hydrostatic pressures in the range of $2900-3350 \mathrm{~cm}^{-1}$.

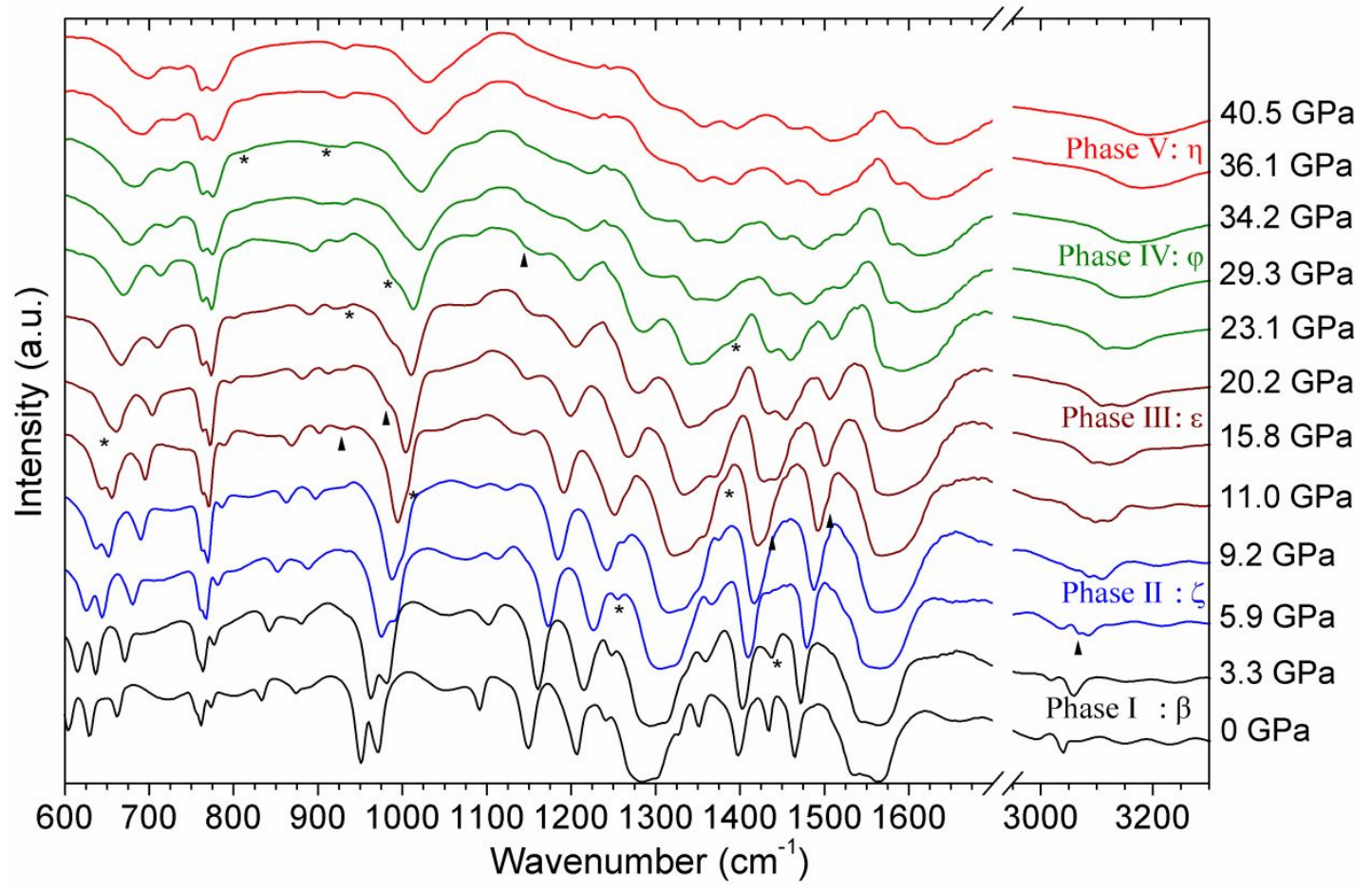

Figure S18 Infrared spectra of HMX under non-hydrostatic pressures with using $\mathrm{KBr}$ as pressure medium. 